\title{
OPTIMIZATION STRUCTURES FOR SUPPLY CHAIN MANAGEMENT
}

\author{
Marcius F. Carvalho ${ }^{1,2}$ and Pythagoras G. Furtado ${ }^{2}$ \\ ${ }^{I}$ National Institute of Information Technology (ITI), Campinas - SP - BRAZIL \\ ${ }^{2}$ Mechanical Engineering Faculty - UNICAMP, Campinas - SP - BRAZIL \\ marcius.carvalho@iti.gov.br; furtado@fem.unicamp.br
}

\begin{abstract}
This paper compares three structures for supply chain production management. To take advantage of the special problem configuration, it models the supply chain planning as a network flow optimization problem. The model considers important aspects as: planning and coordination of activities among enterprises, production capacity constraints, production cost of each enterprise, and demand variation.
\end{abstract}

\section{INTRODUCTION}

A supply chain (SC) is a complex manufacturing system composed of a network of enterprises (suppliers, manufacturers, assemblers, distributors, warehouses) collectively responsible for the procurement, manufacturing and distribution activities associated with one or more families of related products (Reid, 1999). The success of a supply chain depends on the information, material and control flows coordination among enterprises. The coordination is possible through the implementation of a decision-making mechanism considering the objectives of the chain and, at the same time, constraints and objectives of every member of the SC.

To deal with this problem, Carvalho and Campos (1997) suggest a two level management hierarchy to support the decision making process. Through this approach the production planning occurs in two levels: cooperative and enterprise. The cooperative level considers a broad vision of the chain's production process and generates strategic decisions, transformed into targets, to be pursued at the enterprise level. The strategic decisions define the best working point for the chain. The cooperative solution space has to consider the objectives and constraints of each enterprise and, at the same time, the business process among enterprises.

At the second level, the enterprise level, each partner enterprise plans its production having as a target the strategic decision taken at the cooperative level. If some target is not acceptable or intangible, new constraint expressing this fact need to be generated and incorporated at the co-operative level, that will establish new goals. The feedback process eliminates inconsistency and contributes to the planning convergence. The two levels planning structure and the information flow exchange between the cooperative and enterprise levels are shown in the Figure 1.

The original version of this chapter was revised: The copyright line was incorrect. This has been corrected. The Erratum to this chapter is available at DOI: 10.1007/978-0-387-35585-6_68 


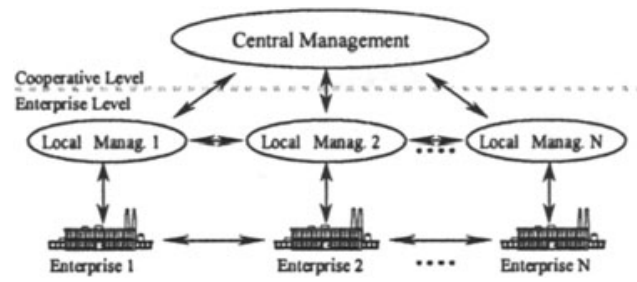

Figure 1-Hierarchical structure for planning

One of the factors that motivates the supply chain hierarchical planning is the information distortion, known as the bullwhip effect (Lee et al., 1997). Forrester (1961), analyzing the flow of goods, information and delays of a production and distribution system, concluded that if the demand of products is transmitted along a series of inventories using stock control ordering, the demand variation increases with each transfer. Simchi-Levi et al.(2000) analyzed the random effect of the customer demand and concluded that the variance of orders grows faster in a decentralized supply chain, compared with a centralized one. In a centralized SC, each enterprise of the chain uses the actual customer demand data to create more accurate forecasts, rather than relying on the orders received form the previous stage, which can vary significantly more than the actual customer demand.

This work studies three management structures for SC production planning. Section two presents the cooperative optimisation model. Section three compares some production planning approaches in a distributed enterprise network and shows the importance of the cooperation among partner enterprises. Finally the conclusion suggests a way to implement a technological structure for cooperation.

\section{SUPPLY CHAIN NETWORK FLOW MODEL}

The cooperative SC production planning has the objective of reaching the customer demand considering the material and capacity availability of every enterprise component of the chain, so that the total production cost brings competitiveness to the final product. Two main structures are discussed below: Serial SC (Figure 2a) and Assembly SC (Figure 2b). Other structures could be obtained combining them.

\subsection{Serial Supply Chain Modelling}

Figure 3a shows the serial supply chain shown in the Figure 2a for three periods of time. Figure $3 \mathrm{~b}$ models the $\mathrm{SC}$ as a network flow optimization problem. Cost and

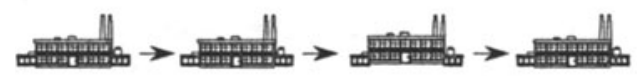

(a)

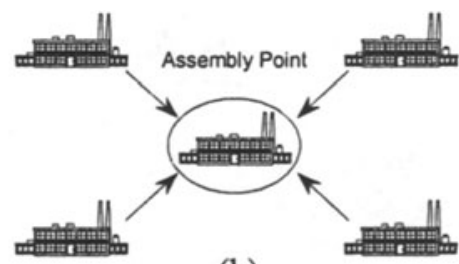

(b)

Figure 2 - Supply chain structures 


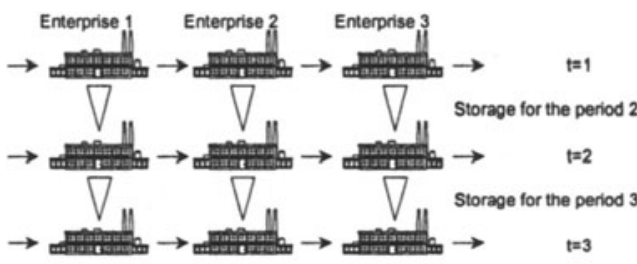

(a)

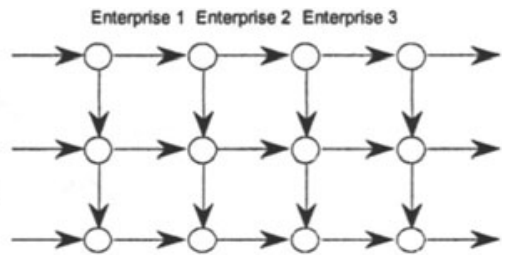

(b)

Figure 3 - Serial supply chain expansion to three time periods

capacity are associated to every enterprise arcs. In the Figure $3 b$, vertical arcs are associated with storage capability between subsequent periods of time, while horizontal arcs represent enterprises. The nodes are decision points where the quantities to be produced or stored are determined. The network flow optimization model, starting from a known demand, to each product family, determines, for each time period, the capacitated planning for each enterprise, raw material requirement, storage policy and good delivery time.

\subsection{Assembly Supply Chain Modelling}

Figure 4 illustrates (a) a two-period assembly chain and (b) its network flow representation. One of the most important management functions in the assembly chain is the coordination of suppliers and assembly enterprises flow. This coordination avoids superfluous storage and production interruptions due to unbalanced production flow.

Carvalho et al. (1999) models the above problem as a multi-period, multiproduct and multi-stage production flow problem adding side constraints to the node-arc constraints matrix. The inclusion of side constraints destroys the network structure of the problem, but their number is not significant, being in the range of $15 \%$ for small problems to $5 \%$ for large problems. This characteristic, added to the block diagonal structure of the problem, motivates the use of a decomposition technique as in Yamakami et al. (2000).
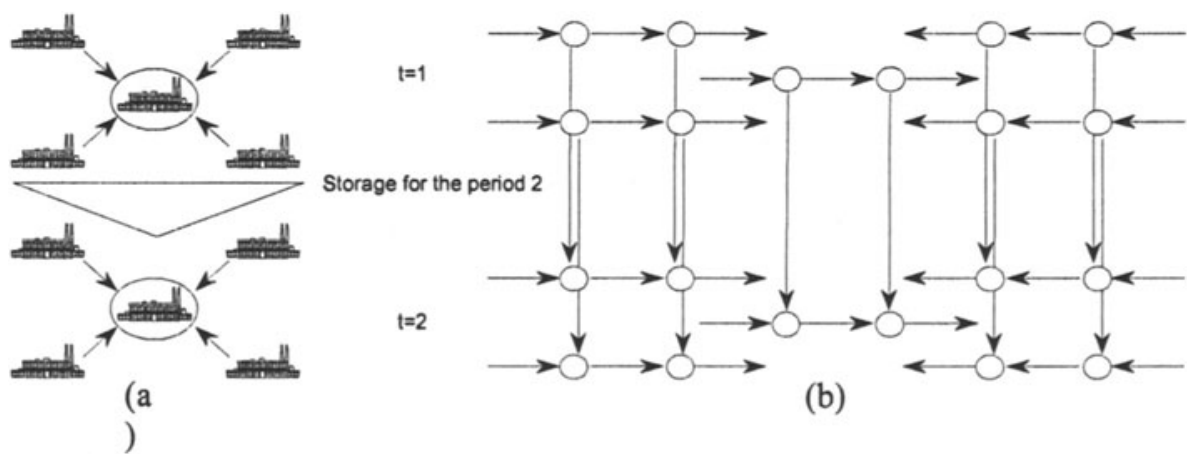

(b)

Figure 4 - Assembly supply chain expansion to two time periods 


\section{CASE STUDY}

A supply chain with four production stages and seven enterprises is shown in Figure 5. The first stage, the retailer stage, has three sale points. They are connected to a distributor that receives the products from an assembler. At the last production stage are two suppliers.

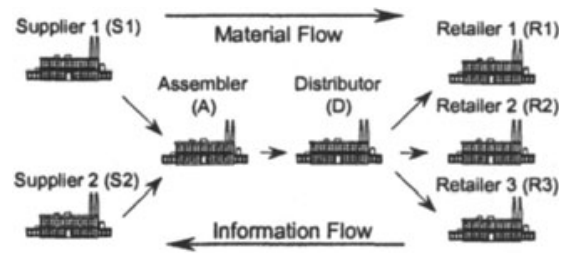

Figure 5 - Supply chain for the case study

This study proposes to analyse the temporal behaviour of the production chain. Three scenarios, where the cooperation between enterprises is progressively increased, are presented. At the first scenario, the supply chain operates as an enlarged JIT structure (Lee and Paek, 1995), where the enterprises optimize their operation based on orders received from their client(s). At the second scenario, partner enterprises directly involved in a business process share detailed temporal demand information. The highest cooperation level is found at the third scenario, where partners share information about demand, capacity and cost.

\subsection{Scenario 1: Local Optimization with JIT Demand Information}

Figure 6 presents the network flow models for the first scenario. The retailer drives the production process starting from known demands $D_{1}, D_{2}$ and $D_{3}$. The retailers plan their operation activities considering their cost and capacity. This plan generates an order to the distributor. The distributor follows the same procedure to
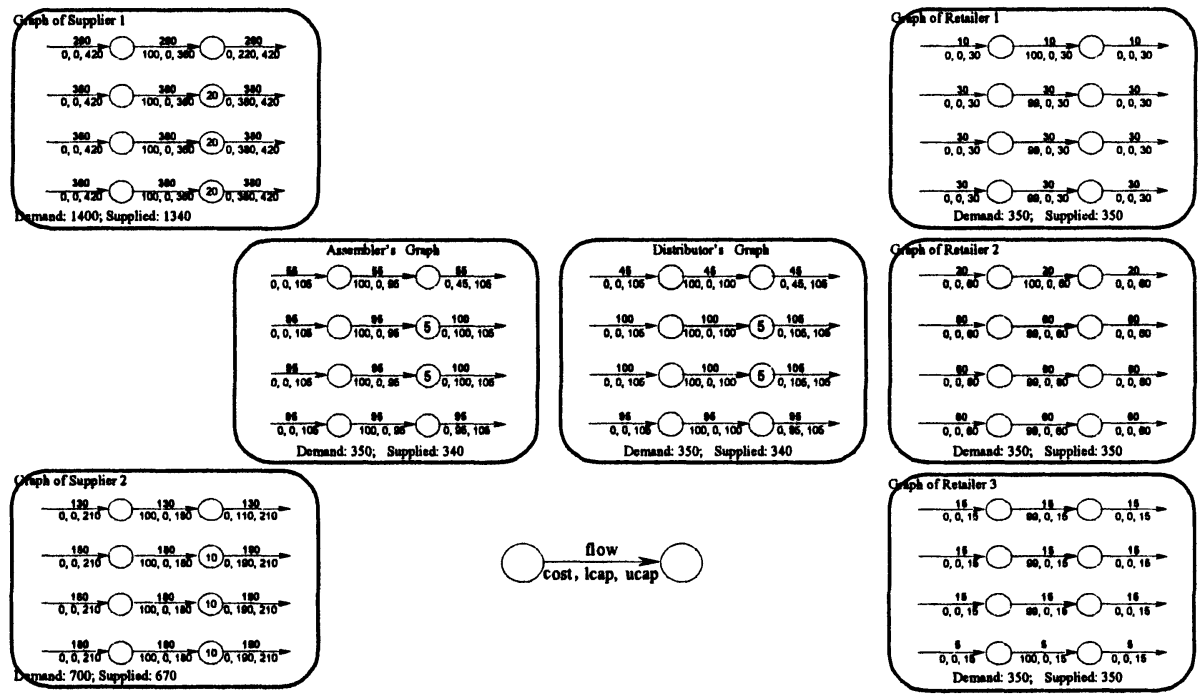

Figure 6-Network flow model of scenario 1 
generate orders to the assembler. Finally, the assembler generates orders to its suppliers.

Three types of arcs are identified in the network flow representation: Raw material arcs, located at the left side of each graph, represent the raw material supply capability and cost of the previous production stage. Production arcs, at the centre of each graph, represent assembly, transformation or transportation processes. Delivery arcs, at the right side of each graph, represent the delivery of final products to the next production stage or to the customer. Figure 6 shows the costs, upper and lower limits, and the optimum production flow. For example, the optimal production policy, for the first retailer is to produce in the weeks 2,3 and 4, since these weeks have lower production cost than week 1 .

Lower capacity limit models orders from the retailers to the distributor (setting the delivery arc capacity limit equal to the retailer's orders). For example, the minimum amount to be delivered by the distributor at week 1 has to be equal to the sum of retailers R1, R2 and R3 orders in this week. Then the lower capacity limit for the distributor delivery arc at week 1 is equal to $\operatorname{prod}(\mathrm{R} 1,1)+\operatorname{prod}(\mathrm{R} 2,1)+$ $\operatorname{prod}(\mathrm{R} 3,1)=10+20+15=45$. Expanding this procedure to week 2, week 3 and week 4 , will result respectively 105,105 and 95 as the lower boundary of the distributor delivery arcs (see the distributor's graph at Figure 6). Note that the monthly production capacity of the distributor (sum of the capacities of the four production arcs $=400$ units) is enough to supply the monthly retailer needs $\left(D_{1}+D_{2}\right.$ $+D_{3}=350$ ). However, the distributor capacities for weeks 2 and 3 (equal to 100 units) are not enough to supply the retailer needs at weeks 2 and 3 (equal to 105 units). Then the difference between the distributor production capacity and the retailer order for these weeks ( 5 units as indicated inside the corresponding nodes) will be lost.

The above capacity problem is extended to the upstream in the chain. Ten more units will not be supplied from the assembler to the distributor due to production limitation of the arcs at weeks 2 and 3. Each unit of assembled product needs 4 units of supplier S1 and 2 units of supplier S2. The JIT production planning approach causes, during weeks 2, 3 and 4,60 units of demand not supplied for the supplier S1 and 30 units for the supplier S2.

The above scenario represents a practical situation for supply chains that follows enlarged JIT approach, with local production planning. The supply receives and makes an effort to reach the buyer orders notwithstanding the production cost. But even with this philosophy, the outcome as shown above, can result in a certain amount of demand not supplied, although the production system would have enough capacity to supply the total demand. The demand not supplied has a high cost for the chain, causing a loss of new business opportunities. This problem makes the partners lose reliability in their suppliers. The low performance of this scenario is related to a lack of cooperation and poor information exchange between partner-enterprises.

\subsection{Scenario 2: Local Optimization by Partial Information Sharing}

This scenario analyzes the supply chain performance when the weekly plan of the buyer-enterprise is known in advance. With this information, the enterprises can anticipate the production and store the products to meet future demand. Vertical arcs 
(called inter-period storage arcs) are introduced to model the storage in the network flow model, as shown in the Figure 7.

The cooperation between neighbour enterprises occurs when: the retailers, starting with the customer demand expectation, define their production planning and their future needs to meet this plan and send immediate order information to the distributor. The distributor anticipates its production in 10 units to the first week, to meet future orders. With this procedure, the distributor can meet the retailer's demand. This anticipation policy uses available capacity for week 1 and stores the exceeding production to deliver to the future needs as shown in the storage arcs of Figure 7. The same cooperation policy can be applied between the distributor and the assembler enterprises and between the assembler and the supplier enterprises. This scenario improves the performance of the supply chain through cooperation and information improvement.
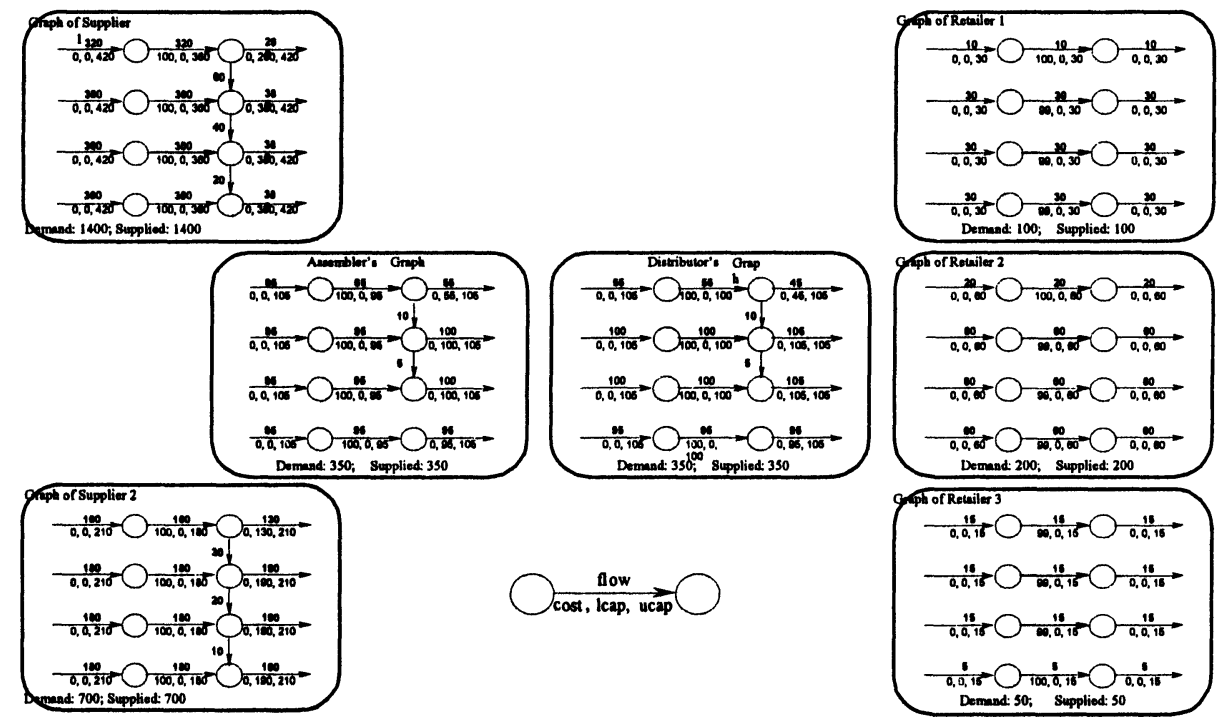

Figure 7 - Network flow model of scenario 2

\subsection{Scenario 3: Global Optimization by Total Information Sharing}

Although scenario 2 improves the system performance observed at scenario 1 , the production networks reached only a rough optimization. This limited result is due to the unconnected networks, shown in Figures 6 and 7 (local optimization). A better supply chain production planning solution can be met by a global optimization, possible by the interconnection of the networks that model the supply chain.

The third scenario considers a strong cooperation among partners, with high information exchange including sharing of production cost and production capacity. The network flow representation for this scenario is presented in the Figure 8. The grey nodes of this figure interconnect the delivery arcs of a selling enterprise to the receiving arcs of a buying enterprise. This simple procedure is applied when the coupling rate between two enterprises is one. To model the interconnection between the assembler and its suppliers, it is necessary to add coupling constraints, as 


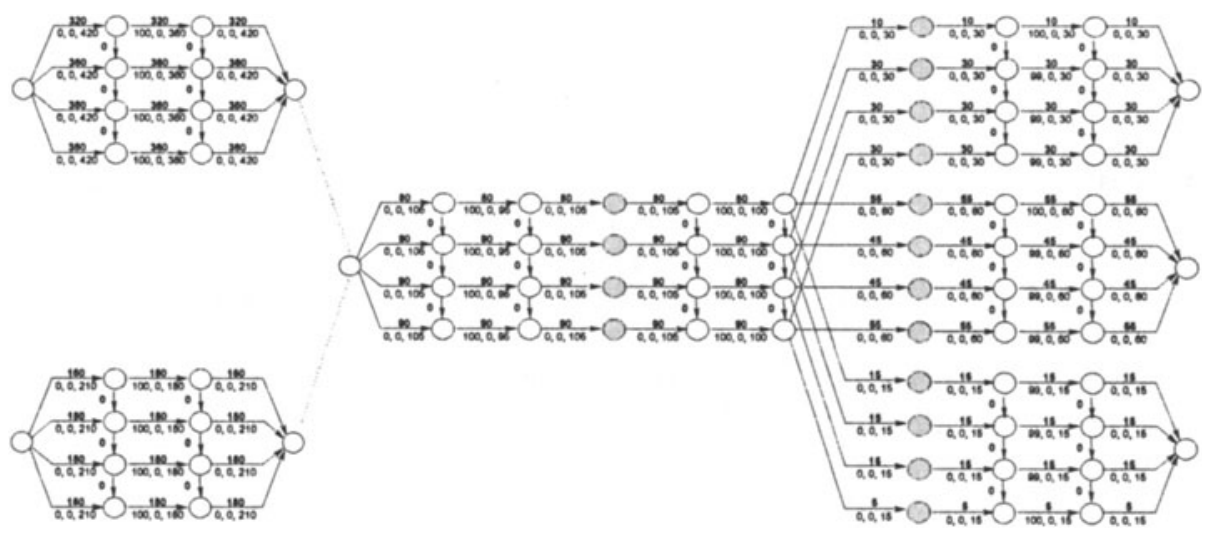

Figure 8-Global network flow model of scenario 3

discussed in section 2 . The coupling constraints synchronize the inter-enterprise production flow. The synchronization constraints of Figure 8 guarantee, for each time interval, that:

- 4 units of part_1 are produced at supplier S1 for each product assembled at the assembly-enterprise;

- 2 units of part_2 are produced at supplier S2 for each product assembled at the assembly-enterprise.

The Table 1 shows the three scenarios results. The analysis of these results shows that the Global Optimization by Total Information Sharing is the best structure to plan an integrated supply chain. It reaches better performance than the previous structures in terms of service quality, backorder level and cost due to the information improvement among partner enterprises. The final scheduling, reached by global optimization, can be proposed as a target or policy to be pursued by every enterprise. The targets have to be seen not as an imposition but as a proposition to be followed by the Supply Chain members.

Table 1- Summary of the results

$(\mathrm{RD}=$ Real demand; $\mathrm{SD}=$ Supplied demand; NSD: Non-supplied demand;

TIS: Total inter-period storage)

\begin{tabular}{|c|c|c|c|c|c|c|c|c|c|c|c|c|}
\hline & \multicolumn{4}{|c|}{ Scenario 1 } & \multicolumn{4}{c|}{ Scenario 2 } & \multicolumn{4}{c|}{ Scenario 3 } \\
\hline Enteprise & RD & SD & NSD & TIS & RD & SD & NSD & TIS & RD & SD & NSD & TIS \\
\hline R1 & 100 & 100 & $\mathbf{0}$ & $\mathbf{0}$ & 100 & 100 & $\mathbf{0}$ & $\mathbf{0}$ & 100 & 100 & $\mathbf{0}$ & $\mathbf{0}$ \\
\hline R2 & 200 & 200 & $\mathbf{0}$ & $\mathbf{0}$ & 200 & 200 & $\mathbf{0}$ & $\mathbf{0}$ & 200 & 200 & $\mathbf{0}$ & $\mathbf{0}$ \\
\hline R3 & 50 & 50 & $\mathbf{0}$ & $\mathbf{0}$ & 50 & 50 & $\mathbf{0}$ & $\mathbf{0}$ & 50 & 50 & $\mathbf{0}$ & $\mathbf{0}$ \\
\hline D & 350 & 340 & $\mathbf{1 0}$ & $\mathbf{0}$ & 350 & 350 & $\mathbf{0}$ & $\mathbf{1 5}$ & 350 & 350 & $\mathbf{0}$ & $\mathbf{0}$ \\
\hline A & 350 & 340 & $\mathbf{1 0}$ & $\mathbf{0}$ & 350 & 350 & $\mathbf{0}$ & $\mathbf{1 5}$ & 350 & 350 & $\mathbf{0}$ & $\mathbf{0}$ \\
\hline S1 & 1400 & 1340 & $\mathbf{6 0}$ & $\mathbf{0}$ & 1400 & 1400 & $\mathbf{0}$ & $\mathbf{1 2 0}$ & 1400 & 1400 & $\mathbf{0}$ & $\mathbf{0}$ \\
\hline S2 & 700 & 670 & $\mathbf{3 0}$ & $\mathbf{0}$ & 700 & 700 & $\mathbf{0}$ & $\mathbf{6 0}$ & 700 & 700 & $\mathbf{0}$ & $\mathbf{0}$ \\
\hline
\end{tabular}




\section{NEGOTIATION AND DATA INTERCHANGE}

Global optimization is only possible through cooperation among partner enterprises, based on the feedback of information between the cooperative planning level and the enterprise level until the convergence of chain production planning.

As suggested by Camarinha-Matos et al. (2000), the negotiation process involves the analysis, for each enterprise, of the strategic decision to be taken, following the importance of the problem. Such a decision can be simple as, for example, the delay of a production activity or the cancellation of one activity. But it also can be complex as the change of a supplier or even the dissolution of the chain. Negotiation based on the centralized information system is the best approach for the supply chain management. It involves:

Data receiving: In this phase the cooperative management system receives data from the enterprises, distributors, retailers and customers expressing their expectation and contribution to the plan as: resources availability, capacity constrains, cost, demand expectation and these data added to the production routes representing the supply chain configuration are the input to the global optimization algorithm. These data are supplied on a blackboard that manages the supply chain information.

Data processing and plan generation: The availability of the above data allows the manager, based on an optimization tool that takes into account the special structure of the problem, to determine production targets to the chain and to each enterprise, defining quantities and time of production that coordinate the flow of products in the chain, in each period of time.

Plan distribution: The cooperative management system has to distribute the above plan to the chain components at a discrete time period. Or as an alternative approach leave the planning information available on the blackboard to allow remote assess at the best time for the partners enterprise but it can generate un-coordination problems.

Planning analysis: The proposed cooperative plan will be feasible to every chain enterprise since the cooperative level considered the enterprises constraints. However, if some enterprises, analyzing these production targets identify inconsistency, due to market dynamics or random events, the enterprise should initially try to negotiate a solution internally, then with its neighborhoods, and finally it has to generate new constraints to be sent to the cooperative level starting a new planning cycle.

The global optimization by total information sharing approach should be supported mainly by communication protocols for information exchange. Several schemas are available in the literature and among them two most interesting for enterprise integration and communication are shown in Diaz and Villemur (1999) and in Mundim and Bremer (2000). 


\section{CONCLUSION}

Enterprises can use local optimization and enlarged JIT approach to improve the supply chain management. Better results can be met by improving information sharing through the communication of future orders to the immediately upstream enterprise. But larger benefit can be obtained by improving the cooperation and collaboration among enterprises within chain particularly through the free exchange of actual demand information and enterprise production capacity. Having a central information sharing is possible to implement a global optimization that generate a production scheduling to be proposed as a target of policy to be pursued to every supply chain members.

In this paper the above approaches for supply chain management were implemented and their performance analysed following a cost and backorder optimisation. This analysis was supported by a case study.

There is at least one more aspect to be considered. It is concerning the question of supply contracts between enterprises, with establishment of production costs and quantities. This question is important, for instance, in the determination of the chain's composition and in the definition of new partners. This is an area with significant opportunities for future research.

\section{Acknowledgements}

The authors thank FAPESP (The State of São Paulo Research Foundation) and CNPq (The Brazilian Council for Research and Scientific Development) for the financial support.

\section{REFERENCES}

1. Camarinha-Matos, L.M., Afsarmanesh, H., Rabelo, R. (2000) Supporting agility in virtual enterprises. In: E-Business and Virtual Enterprises (L.M. Camarinha-Matos, H. Afsarmanesh, R.J. Rabelo (Ed.)). pp.113-126. Kluwer Academic Publishers, Boston.

2. Carvalho, M.F., Campos, R. (1997) A Hierarchy for Cooperative Enterprise. IFAC/IFIP Conference on Managment and Control of Production and Logistics, MCPL, pp 420-425.

3. Carvalho, M.F., Fernandes, C.A.O., Ferreira, P.V. (1999) Multiproduct multistage production scheduling (MMPS) for manufacturing systems. Production Planning \& Control, v.10, n.7, pp.671-681.

4. Diaz, M. and Villemur, T. (1999) A collaborative membership service and protocol for structured groups. LAAS Report. http://www.laas.fr/laasve/index.htm.

5. Forrester, J.W. (1961) Industrial Dynamics. MIT Press, Boston, MA.

6. Lee, H.L., Padmanabhan, V. and Whang, S. (1997) Information distortion in a supply chain: the bullwhip effect. Management Science, 43, 546-558.

7. Lee, S.M. and Paek, J.H. (1995) An enlarged JIT programme: its impact on JIT implementation and performance of the production System. Production Planning \& Control, v.6, n.2, pp185-191.

8. Mundim, A.P. and Bremer, C.F. (2000) Design of a Computer-Supported Cooperative Environment for Small and Medium Enterprises. In: E-Business and Virtual Enterprises (L.M. CamarinhaMatos, H. Afsarmanesh, R.J. Rabelo (Ed.)). pp.113-126. Kluwer Academic Publishers, Boston.

9. Reid, R.L. (1999) Engineering the Virtual Enterprise. http://arrirs04.uta.edu:80/eif/ve_ie.pdf. 
10. Simchi-Levi, D., Kaminsky, P. and Simchi-Levi, E. (2000) Designing and Managing the Supply Chain: Concepts, Strategies, and Cases. McGraw-Hill.

11. Yamakami, A., Takahashi, M., Carvalho, M. (2000) Comparison of Some Algorithms for Manufacturing Production Planning. IFAC-MIM 2000 Symposium, pp.280-285. 